\title{
Corrigendum
}

\section{Corrigendum to "Ultrafast Probe of Carrier Diffusion and Nongeminate Processes in a Single CdSSe Nanowire"}

\author{
Peter S. Eldridge, ${ }^{1}$ Jolie C. Blake, ${ }^{1}$ and Lars Gundlach ${ }^{1,2}$ \\ ${ }^{1}$ Department of Chemistry and Biochemistry, University of Delaware, Newark, DE 19716, USA \\ ${ }^{2}$ Department of Physics and Astronomy, University of Delaware, Newark, DE 19716, USA \\ Correspondence should be addressed to Lars Gundlach; larsg@udel.edu \\ Received 11 September 2015; Accepted 27 September 2015
}

Copyright (C) 2015 Peter S. Eldridge et al. This is an open access article distributed under the Creative Commons Attribution License, which permits unrestricted use, distribution, and reproduction in any medium, provided the original work is properly cited.

In the paper titled "Ultrafast Probe of Carrier Diffusion and Nongeminate Processes in a Single CdSSe Nanowire" [1], the Acknowledgment section was missing. Here we added this section.

\section{Acknowledgment}

Acknowledgment is made to the donors of the American Chemical Society Petroleum Research Fund for partial support of this research.

\section{References}

[1] P. S. Eldridge, J. C. Blake, and L. Gundlach, "Ultrafast probe of carrier diffusion and nongeminate processes in a single CdSSe nanowire," Journal of Spectroscopy, vol. 2015, Article ID 574754, 6 pages, 2015. 

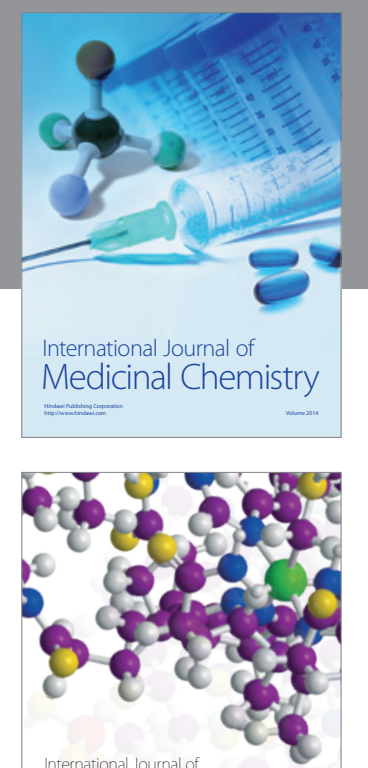

\section{Carbohydrate} Chemistry

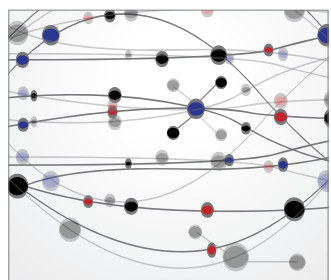

The Scientific World Journal
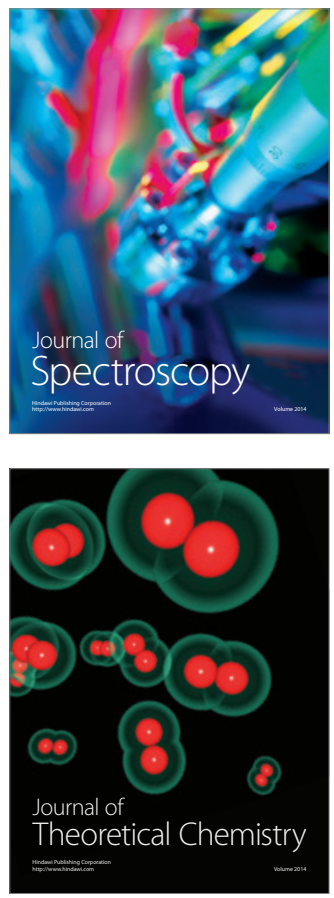
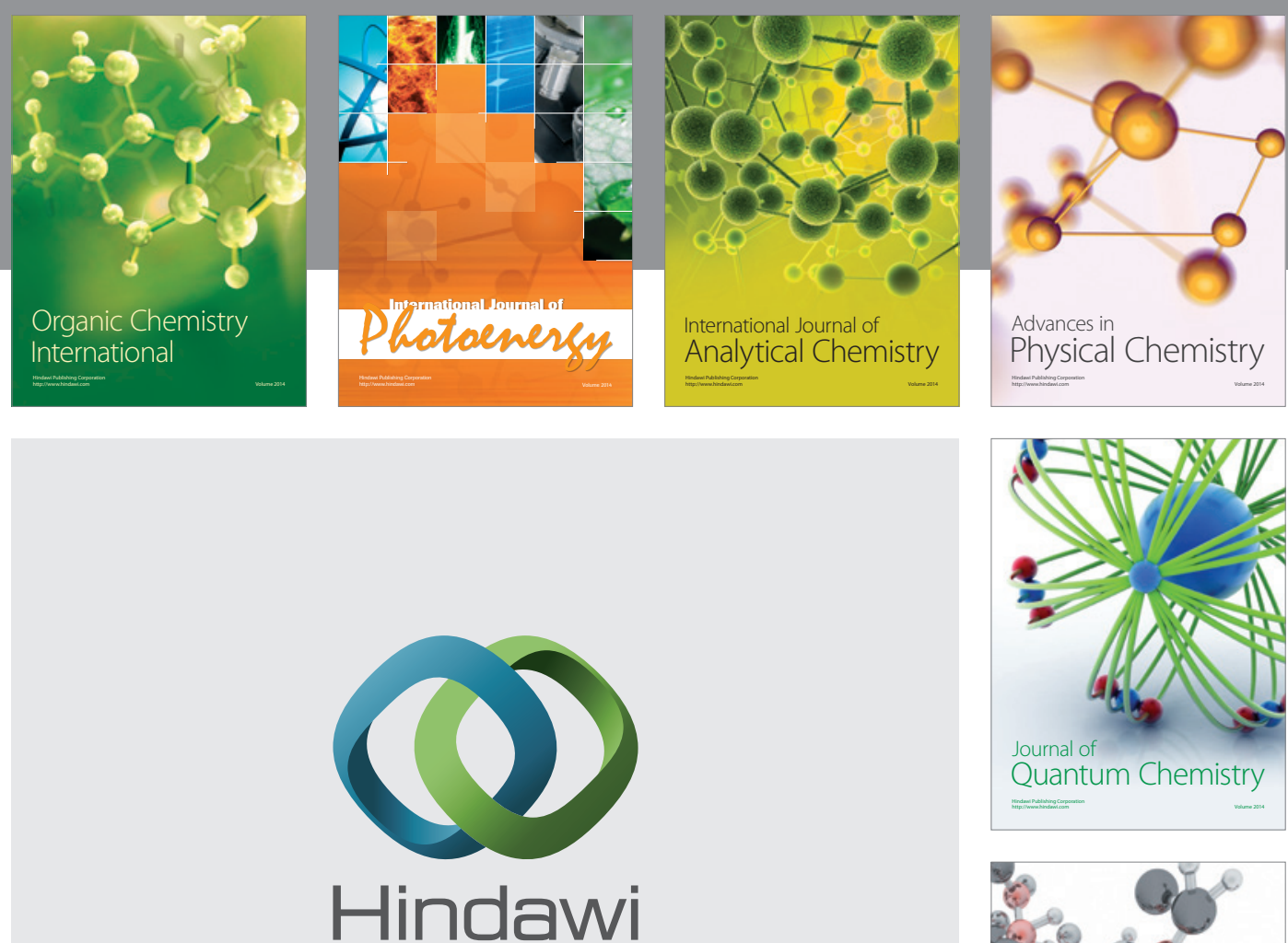

Submit your manuscripts at

http://www.hindawi.com

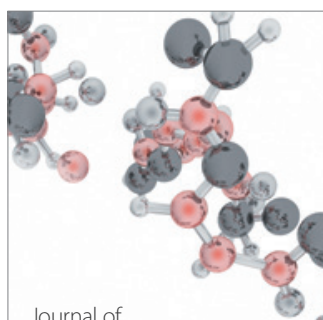

Analytical Methods

in Chemistry

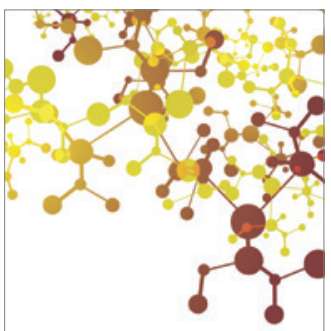

Journal of

Applied Chemistry

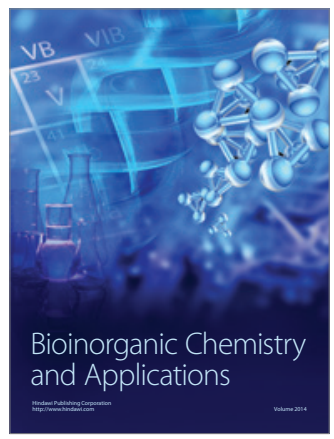

Inorganic Chemistry
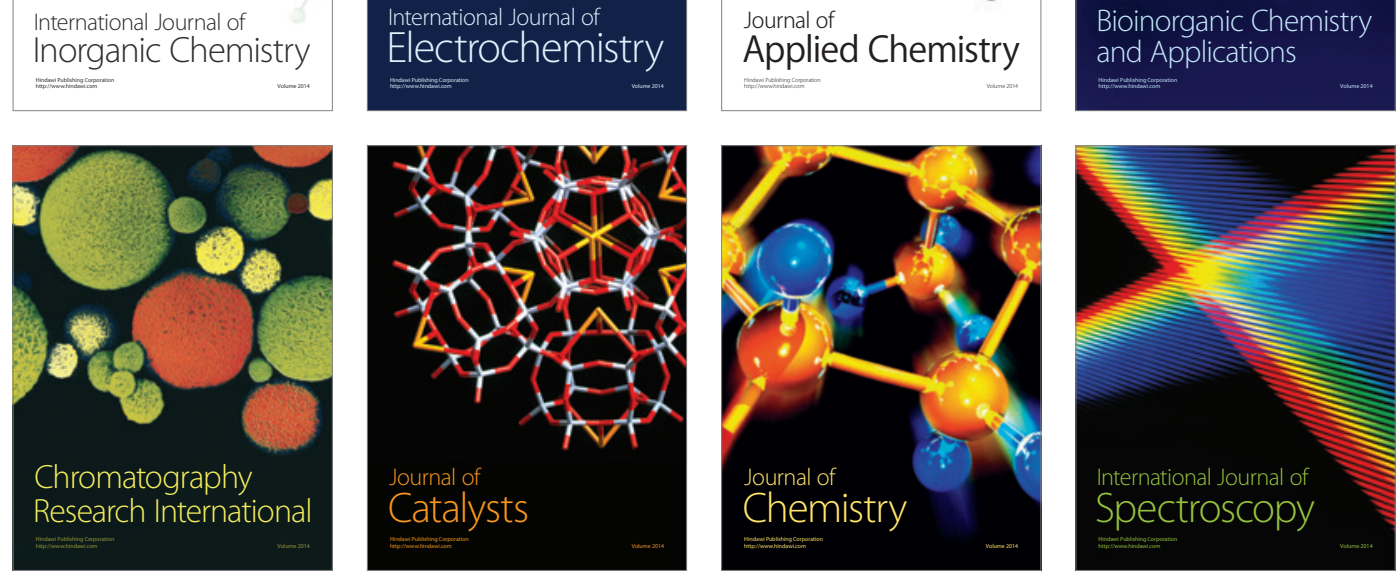